\title{
Bend sensor arrays for hand movement tracking in biomedical systems
}

\author{
Giovanni Saggio \\ Dept. of Electronic Engineering, University of Tor Vergata \\ Via del Politecnico 1, 00133 Rome, Italy \\ saggio@uniroma2.it
}

\begin{abstract}
Bend sensor's electrical feature and mechanical pliability allow to easily realize a cost effective and reliable system to measure human postures. With this kind of sensors we realized a data glove capable to accurately measure all degree of freedom of a human hand. In particular, in collaboration with the Flexpoint Sensor Systems Inc., we designed and realized a unique device capable to measure at the same time the flex-extension movements of the three joints of one finger. Experimental results demonstrated good performances obtained in terms of accuracy and repeatability of the measures.
\end{abstract}

\section{Background}

Bend sensors are successfully applied in many fields such as automotive (pedestrian impact detection, seat occupant detection, horn switch, etc.) [1], industrial (automated manufacturing controls, robotics, safety switches, quality control, inspection controls, shipping and handling applications and machine control) [2], consumer toys (product applications that emphasize functionality, reliability, quality, and user convenience) [3], musical (to play using body movements and basis for note transcription systems) [4], medical (in infusion pumps, respiratory therapy equipment, obstetric equipment, rehabilitative and physical therapy equipment, pelvic stabilization devices, Pressure-Monitoring Devices, etc.) [5]. Among all, it is in particular in the medical field that we focused our efforts adopting these kind of sensors to produce a data glove for rehabilitation purposes. In fact it is currently adopted at the Hand Surgery Department of the Hospital of Belcolle in Viterbo (Italy) [6].

\section{Bend sensors}

A bend sensors is nowadays the thinnest, lightest, most robust and cost-effective variable resistor available. It is selfcontained and requires no mechanical components, so the noise normally associated with mechanical swiping and rubbing are avoided. Furthermore, this sensor is not prone to degradation through mechanical contact, so it has a sufficient application life for the most part of the possible applications.

The bend sensor potentiometer consists of a coated substrate, such as plastic, that changes in electrical resistivity when bent. Electronic system can be than connected to the sensor so to acquire data relative to the amount of bending that occurs.

\section{Materials and Methods}

In order to measure the flex-extension movements of a finger joint, it is necessary to lay a bend sensor on that joint. So, in principle, we need one single sensor for one single joint, 15 sensors for 15 joints in total (see Fig. 1). So, the usual way of proceeding is to sew 15 pockets on a support glove to house the 15 sensors. But this common simple approach can offer some drawbacks.

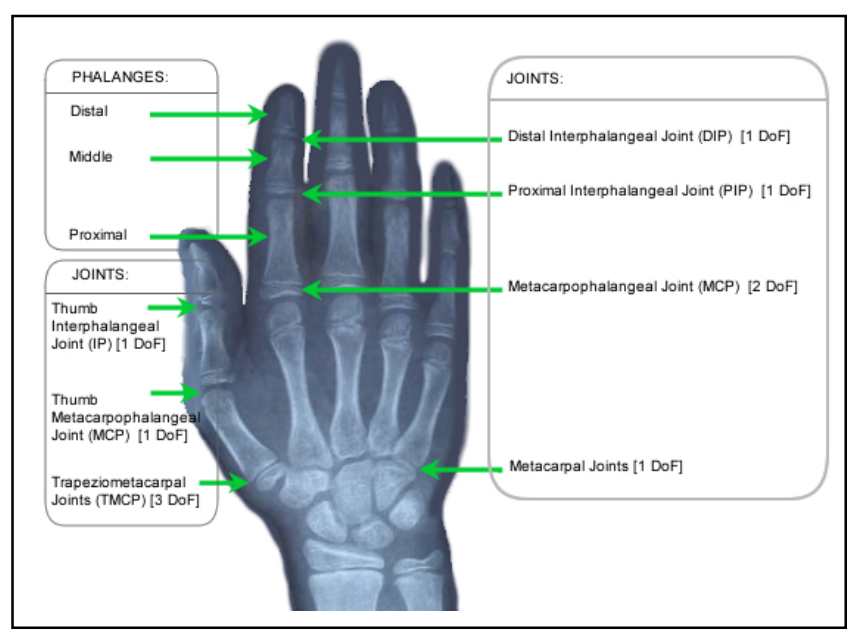

Figure 1: The 15 joints in a human hand

In fact, in this way the necessary perfect alignment between each sensor with its relative joint could not be always assured and maintained. In addiction from each tip of each sensor, two connection wires must be welded (see Fig. 2 ), so to manage 30 wires in total could be another disadvantage. This is because these wires can be subjected to be torn and can restrict the free of movements of the hand. Even if we sewn these wires with the glove, in any case it is difficult to find a "path" that does not obstruct the necessary free movements of the sensors. In fact a sensor must be capable to slide while the joint bends to avoid to be subjected to elongation forces.

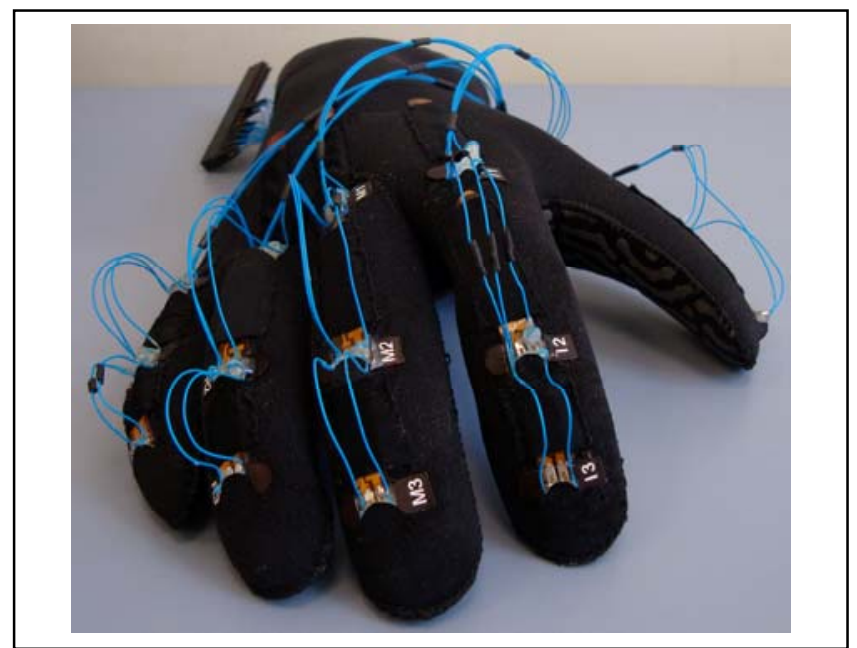

Figure 2: Sensors and wires applied on a support glove 
For these reasons here we propose a different approach to the problem. In collaboration with the Flexpoint Sensor Systems Company, we designed and realized a sensor array. It consists of a unique substrate on top of which three sensible elements are realized (black areas in Fig. 3).

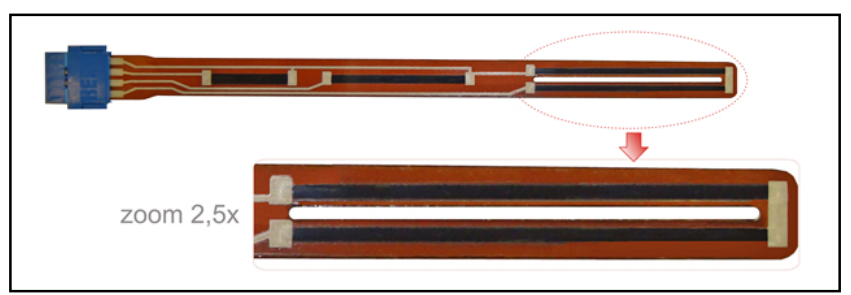

Figure 3: Sensor array. One area is detailed

As the previous figure shows, this array provides three sensors, one of which is designed with a slot running in the central part of the sensor itself (zoomed part of Fig. 3). So, if a tip is fixed in a specific area on the dorsal part of the hand and is inserted into the slot, it becomes a sort of rail for the movement of the entire array (Fig. 4). Moreover, in correspondence with the sensor \#3, the end of the array is maintained fixed with the tip of the finger. In this way the problem of misalignment is completely avoided.

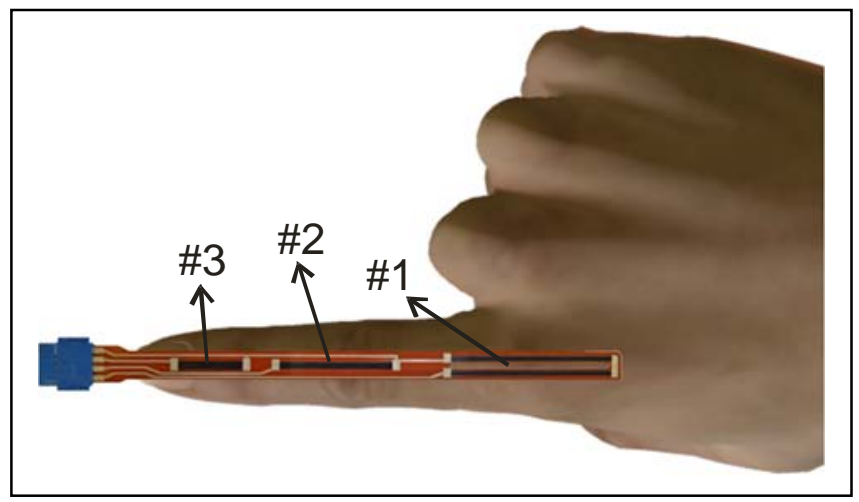

Figure 4: One sensor array on top of the index finger

The previously mentioned problem of the wires is minimized too. In fact, all the electrical terminals are lodged together and reduced from 6 to only 4 since three are in common (the ground), all the connections are realized on the tip of the finger, and the wires can run united across the palm of the hand, without being a problem for the dorsal part of the fingers.

The array was design also with the prevision of realizing a unique substrate capable to lodge in a sort of "matrix" all the sensors for a whole hand, so to obtain a compact structure and with all the wires connected to only one border of the unique sensor's substrate.

\section{Array characterization}

Before the proper utilization of the sensor array to measure the human hand postures, we proceeded with an offline characterization. We wanted to obtain the expression of the function relative to the electrical resistance value variations vs. the amount of bending of the sensors. To this aim we realized a specific set-up capable to mimic the possible flex-extension finger's joint movements. The first step was to design a system capable to simulate the bending positions of one single finger's joint. So we realized a hinge, made of a knuckle through which a central circular pin is passed, and with two notched leafs which extend laterally from the knuckle. One of the leaf is fixed with the pin, so capable to revolve together it, while the other one is maintained fixed. Three of these hinges were joined together, as depicted in Fig. 5a. Three stepper motors were then singularly connected with each central pin of the three hinges. These latter were cross-related, in a multiple pin hinges layout, so that the movements of one leaf of one hinge is capable to translate the complete arrangement of the other two hinges together with their stepper motors (see Fig. 5b).

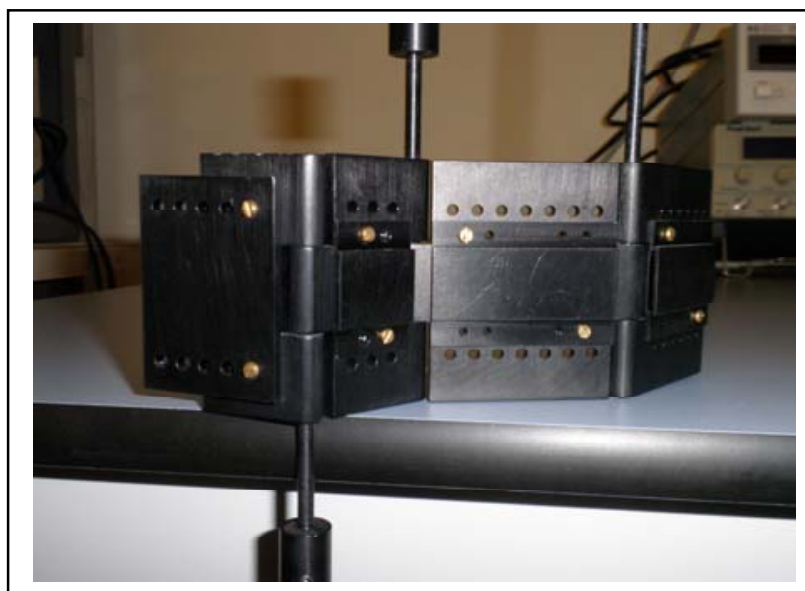

(a)

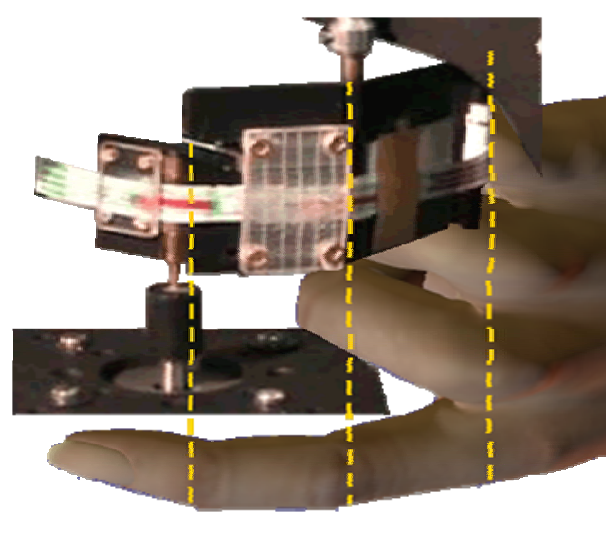

(b)

Figure 5: three hinges set-up

All measurements were performed from $0^{\circ}$ (flat position for the sensor) to $90^{\circ}$, with a step of $10^{\circ}$, iterating 10 times, with 10 acquisitions of the resistance values for every step.

Our question was if a unique substrate could influence the electrical behavior of the sensors, i.e. if three sensors alone could offer an electrical characteristic behaviors differing from the ones of three sensors on the same substrate. This is to investigate if an unique substrate could introduce mechanical problems which could affect the total electrical performances. 


\section{Discussion and Conclusions}

The answer can be obtained analyzing the graph reported in Fig. 6. There are represented the results of a complete $0^{\circ} \div 90^{\circ}$ sweep, stepped $10^{\circ}$, of the sensor $\# 1$, the one nearest to the dorsal part of the hand, measured every $10^{\circ}$ of bending of the sensor \#2, the one in the middle (sensor 1 vs. 2). The results are comparable to those known for one sensor alone (see Ref. [7]). Only a very little shift of the resistance value must be taken into account for higher bending degree which, in any case, is negligible for the most part of applications.

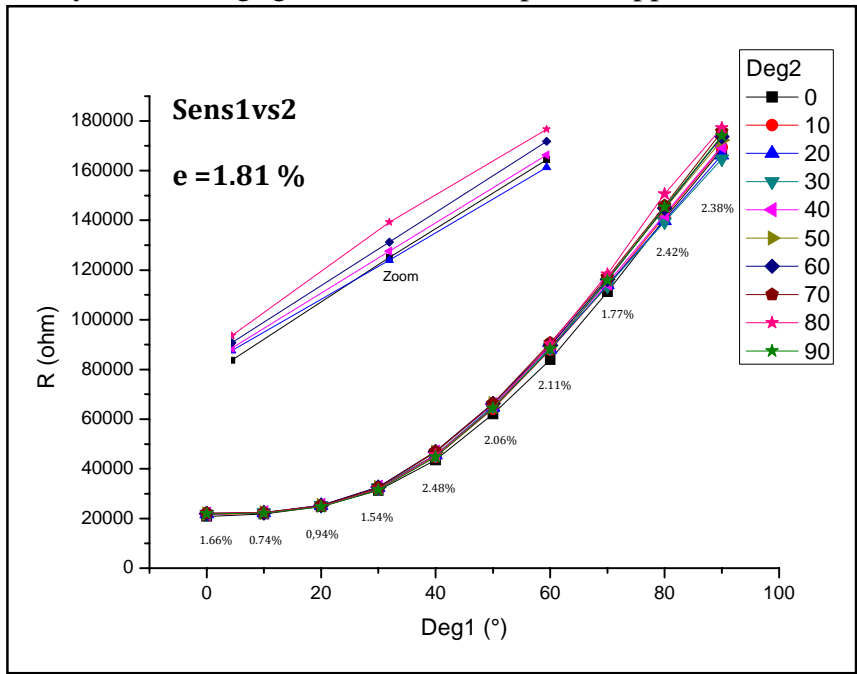

Figure 6: Every $10^{\circ}$ of bending angle for the sensor \#2, a complete sweep between $0^{\circ}$ and $90^{\circ}$ is made for the sensor \#1

Similarly, in Fig. 7 is represented the electrical characteristic within $0^{\circ} \div 90^{\circ}$ angles of the sensor \#1, measured every $10^{\circ}$ of bending of the sensor \#3 (sensor 1 vs. 3).

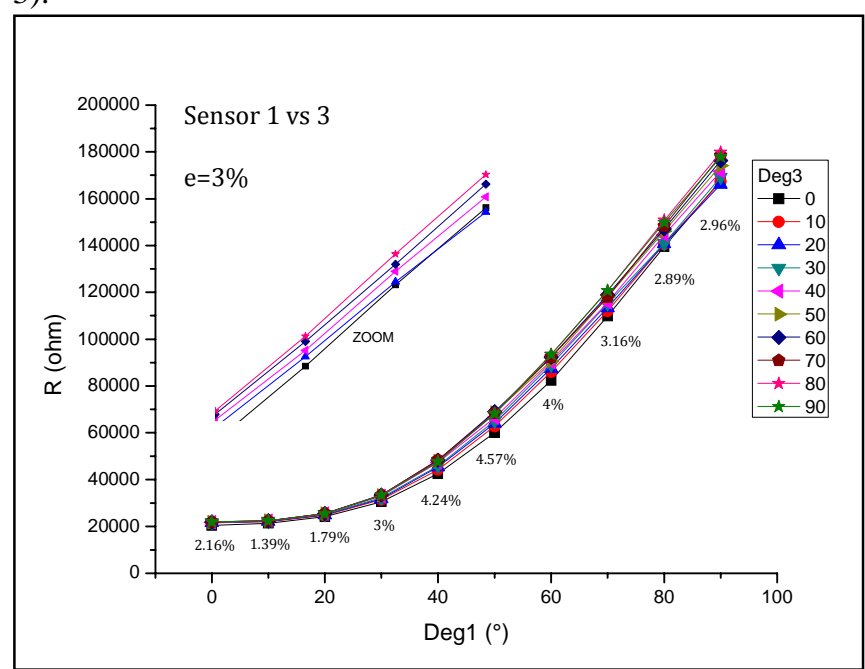

Figure 7: Every $10^{\circ}$ of bending angle for the sensor \#3, a complete sweep between $0^{\circ}$ and $90^{\circ}$ is made for the sensor \#1

These latter results, and all the possible others (sensor 2 vs. 1 , sensor 3 vs. 1 ) were demonstrated to be similar. A slight exception was find in the sensor 2 vs. 3 characterization (Fig. 8) for which the resistance shift is more evident. The reasons for this result are under investigation but, in any case, it is not difficult to take into account this aspect providing for a correction algorithm in the data analysis phase.

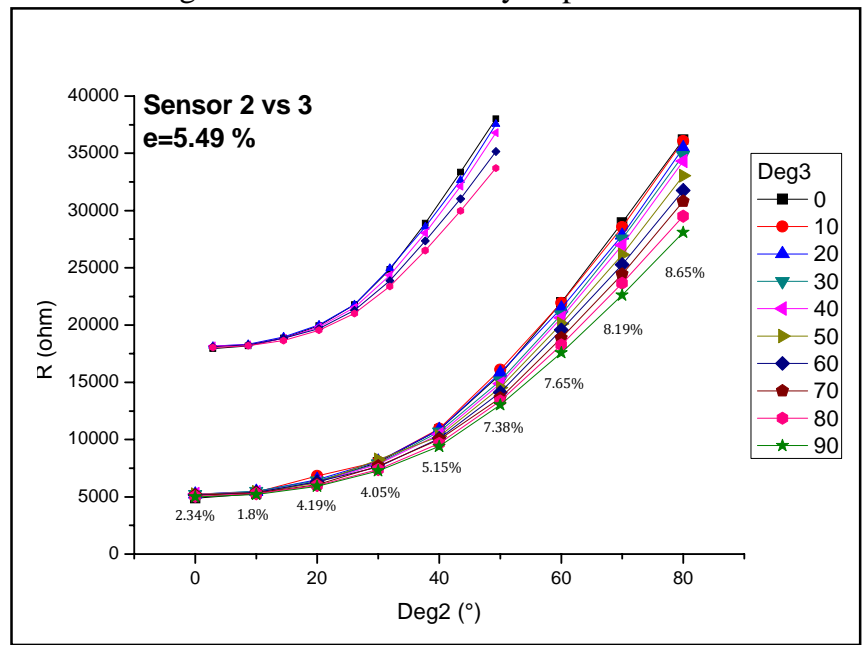

Figure 8: Every $10^{\circ}$ of bending angle for the sensor \#3, a complete sweep between $0^{\circ}$ and $90^{\circ}$ is made for the sensor \#2

In conclusion here we proposed a sensor array devoted to the measure of the finger's joint movements of a human hand. The experiments demonstrated good performances obtained in terms of accuracy and repeatability of the measures. The sensor array can be then successfully adopted to solve some practical problem related to the measure when performed adopting single bend sensor, as usually done.

\section{References}

1. Stanley J.G., Tran, V.H, "Seat belt tension measurement device using a bend sensor," United States Patent US5965827, Oct 12, 1999

2. Kure K., Kanda T., Suzumori K., Wakimoto S., "Flexible displacement sensor using injected conductive paste," Sensors and Actuators A 143 (2008) 272-278

3. Eng M.W., "FlexM: A Computational Construction Kit for Architectural Modeling," Thesis, Master of Architecture, University of Washington, 2004

4. Costantini G., Todisco M., Saggio G. "On the Effect of Memory Width in Automatic Transcription Systems for Polyphonic Piano Music," The 2nd International MultiConference on Complexity, Informatics and Cybernetics: IMCIC 2011, March 2011 Orlando, Florida USA

5. Jurgens J., Patternson P.E., “Development and evaluation of an inexpensive sensor system for use in measuring relative finger positions,” Med. Eng. Phys. Vol. 19, No. 1, pp 1-6, 1997

6. Castagnaro A., De Leo A., Inciocchi S., Saggio G., Tarantino U., "Nuova biotecnologia: il guanto sensorizzato per misurare la cinesi della mano," $48^{\circ}$ Congresso Nazionale Società Italiana Chirurgia della Mano SICM 2010Savona 29 Settembre - 2 Ottobre, 2010

7. Saggio G., Bocchetti S., Pinto C.A., Orengo G., "Wireless Data Glove System developed for HMI,” ISABEL2010, 3rd International Symposium on Applied Sciences in Biomedical and Communication Technologies, Rome, Italy, November 7-10, 2010 